\title{
An Overview of Wireless Indoor Geolocation Techniques and Systems
}

\author{
Kaveh Pahlavan, Xinrong Li, Mika Ylianttila, Ranvir Chana, and Matti Latva-aho \\ Center for Wireless Information Network Studies, Worcester Polytechnic Institute, USA \\ \{kaveh, xinrong\}@ece.wpi.edu \\ Centre for Wireless Communications, University of Oulu, Finland \\ \{over, rschana, matla\} eees 2 .oulu.fi
}

\begin{abstract}
Wireless indoor networks are finding their way into the home and office environments. Also, exploiting location information becomes very popular for both wireless service providers and consumers applications. However, the indoor radio channel causes challenges in extracting accurate location information in indoor environment so that traditional GPS and cellular location systems cannot work properly in indoor areas. This paper provides an overview of the indoor geolocation techniques. After introducing an overall architecture for indoor geolocation systems, technical overview of two indoor geolocation systems are presented. To demonstrate the predicted performance of such systems some simulation results obtained from an indoor geolocation demonstrator are presented.
\end{abstract}

\section{Introduction}

Today numerous wireless indoor products such as cordless telephone, wireless security systems, cordless speakers and even wireless Internet access have been introduced to the consumers. The same way as in late 70's and early 80's the increasing number of terminals in the offices initiated the LAN industry, today increasing number of wireless terminals in indoor areas is promoting wireless indoor networking. Indoor areas are difficult for wiring and most people are reluctant to allow workers to do extensive wiring inside buildings. Wireless is a cost efficient solution that can also provide additional feature of mobility and convenient relocatability. These reasons leave wireless as the preferred medium for indoor networking in the future and promote local networking activities such as Bluetooth, Home-RF, IEEE 802.11 and HIPERLAN/2.

An important evolving technology in recent years has been the indoor geolocation technology both for military and commercial applications. In the commercial application there is an increasing need for these systems for application in hospitals to locate patients or expensive equipment and in homes to locate children and equipment. Military and public safety applications in urban scenarios have promoted a need for inbuilding communication and geolocation networks enabling soldiers, policeman, and fire fighters to complete their missions in urban areas. These incentives have lead to research in indoor geolocation systems [1] 2] 3]. Due to indoor path loss, traditional GPS or E-911 location system cannot work properly in 
indoor environment. As a result, dedicated indoor geolocation systems have to be developed to provide accurate indoor geolocation services.

This paper provides an update on the trends in indoor geolocation systems. In section 2, we briefly discuss the overall system architecture and various geolocation metrics that can be used in indoor environment. Then technical overviews of two indoor geolocation products are presented in Section 3. In Section 4, some simulation results obtained from an indoor geolocation testbed are included. Finally, we close this paper with a short conclusion.

\section{Wireless Geolocation Methods and Metrics}

Most of the geolocation system architectures and methods developed for cellular systems are applicable for indoor geolocation systems although special considerations are needed for indoor radio channels. The most widely used wireless geolocation metrics include Angel of Arrival (AOA), Time of Arrival (TOA), Time Differences of Arrival (TDOA), Received Signal Strength (RSS) and Received Signal Phase. In this section we present an overview of overall system architectures and basic concepts of geolocation metrics as well as corresponding geolocation methods. The possibility of using these methods in indoor environment is also considered.

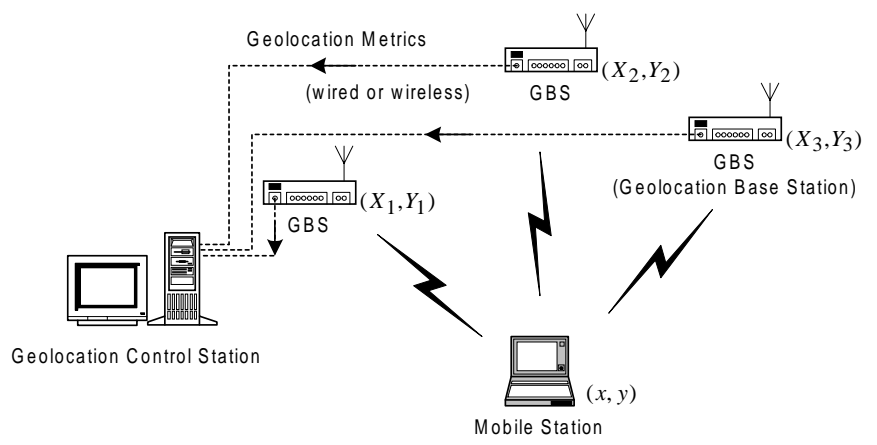

Fig. 1. Overall architecture of indoor geolocation system.

\subsection{Overall System Architecture}

Similar to the cellular geolocation system, the architecture of indoor geolocation systems can be roughly grouped into two main categories: mobile-based architecture and network-based architecture. Most of the indoor geolocation applications proposed to date have been focused on network-based system architecture as shown in Fig. 1 [4 5]. The geolocation base stations (GBS) extract location metrics from the radio signals transmitted by the mobile station and relay the information to a geolocation control station (GCS). The connection between GBS and GCS can be either wired or wireless. Then the position of the mobile station is estimated, 
displayed and tracked at the GCS. With the mobile-based system architecture, the mobile station estimates self-position by measuring received radio signals from multiple fixed GBS. Compared to mobile-based architecture, the network-based system has the advantage that the mobile station can be implemented as a simplestructured transceiver with small size and low power consumption that can be easily carried by people or attached to valuable equipments as a tag.

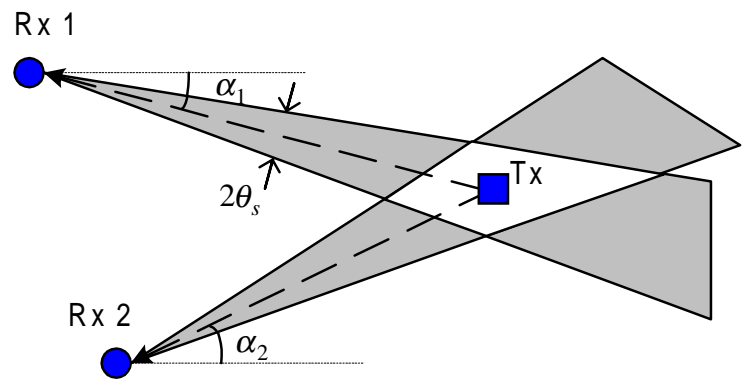

Fig. 2. Angel of Arrival geolocation method.

\subsection{Angle of Arrival}

The AOA geolocation method uses simple triangulation to locate the transmitter as shown in Fig. 2. The receiver measures the direction of received signals (i.e. angel of arrival) from the target transmitter using directional antennas or antenna arrays. If the accuracy of the direction measurement is $\pm \theta_{s}$, AOA measurement at the receiver will restrict the transmitter position around the line-of-sight (LOS) signal path with an angular spread of $2 \theta_{s}$. AOA measurements at two receivers will provide a position fix as illustrated in Fig. 2. We can clearly observe that given the accuracy of AOA measurement, the accuracy of the position estimation depends on the transmitter position with respect to the receivers. When the transmitter lies between the two receivers, AOA measurements will not be able to provide a position fix. As a result, more than two receivers are normally needed to improve the location accuracy. For macro-cellular environment where the primary scatters are located around the transmitter and far away from the receivers, AOA method can provide acceptable location accuracy [6] But dramatically large location errors will occur if the LOS signal path is blocked and the AOA of a reflected or a scattered signal component is used for estimation. In indoor environment, the LOS signal path is usually blocked by surrounding objects or walls. Thus AOA method will not be usable as the only metric for indoor geolocation system.

\subsection{Time of Arrival and Time Difference of Arrival}

The TOA method is based on estimating the propagation time of the signals (i.e. TOA) from a transmitter to multiple receivers. Several different methods can be used 
to obtain TOA or TDOA estimates, including pulse ranging [9 10], phase ranging [9] and spread-spectrum techniques [4] 11]

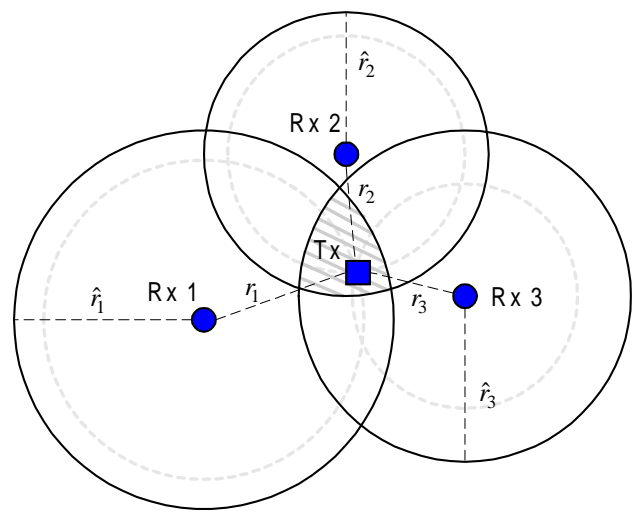

Fig. 3. Time of Arrival geolocation method.

Once TOA is measured, the distance between the transmitter and receiver can be simply determined since the propagation speed of the radio signal is approximately the speed of light $c=3 \times 10^{8} \mathrm{~m} \mathrm{~s}^{-1}$. The estimated distance at the receiver will geometrically define a circle, centered at the receiver, of possible transmitter positions. TOA measurements at three receivers will provide a position fix and given receiver coordinates and distances from the transmitter to receivers, the transmitter coordinates can be easily calculated. Due to multipath propagation, no-line-of-sight (NLOS) signal path and other impairments, the TOA-based distance estimates are always larger than the true distance between the transmitter and the receiver as illustrated in Fig. 2 where $\hat{r}_{1}, \hat{r}_{2}$ and $\hat{r}_{3}$ are the estimated distances and $r_{1}, r_{2}$ and $r_{3}$ are the true distances. Three TOA measurements determine a region of possible transmitter position as shown in Fig. 2. A nonlinear least square (NL-LS) method is usually used to obtain the best estimation iteratively by minimizing the estimation errors [6] 4]:

$$
e_{i}(x, y)=\hat{r}_{i}-\sqrt{\left(X_{i}-x\right)^{2}+\left(Y_{i}-y\right)}
$$

for $i=1,2, \ldots, N$ where $\left(X_{i}, Y_{i}\right)$ are receiver coordinates and $(x, y)$ is transmitter coordinates. Sometimes the transmission time $t_{0}$ of the signals at the transmitter is also taken into account as the third variable:

$$
e_{i}\left(x, y, t_{0}\right)=c\left(t_{i}-t_{0}\right)-\sqrt{\left(X_{i}-x\right)^{2}+\left(Y_{i}-y\right)}
$$

where $t_{i}$ is the receiving time of the signal at the $i$-th receiver. A constrained NL-LS algorithm is also available which makes use of the fact that TOA-based distance estimates are always larger than the true distance [8]. The same as in AOA method, more than three TOA measurements are needed to improve the accuracy of position estimation. 
Instead of using TOA measurements, time difference measurements can also be employed to locate the receiver position. A constant time difference of arrival (TDOA) for two receivers defines a hyperbola, with foci at the receivers, on which the transmitter must be located. Three or more TDOA measurements provide a position fix at the intersection of hyperbolas. NL-LS method can also be used to obtain the best estimation of the transmitter position by minimizing the estimation error:

$$
e_{i, j}(x, y)=c \hat{\tau}_{i, j}-\left[\sqrt{\left(X_{i}-x\right)^{2}+\left(Y_{i}-y\right)^{2}}-\sqrt{\left(X_{j}-x\right)^{2}+\left(Y_{j}-y\right)^{2}}\right]
$$

for $i, j=1,2, \ldots, N$ where $\hat{\tau}_{i, j}$ is the TDOA measurement of $i$ th and $j$ th receivers. There are some other methods to solve the hyperbolic position estimation problem as proposed in [12] [13] [14] and [15]. Compared to TOA method, the main advantage of TDOA method is that it does not require the knowledge of the transmit time from the transmitter while TOA method requires. As a result, strict time synchronization between transmitter and receivers is not required. However, TDOA method requires time synchronization among all the receivers.

\subsection{Received Signal Strength}

If the power transmitted by mobile terminal is known, measuring received signal strength (RSS) at receiver will provide the distance between the transmitter and the receiver using a known mathematical model for radio signal path loss with distances. The same as in the TOA method, the measured distance will determine a circle, centered at the receiver, on which the mobile transmitter must lie. Three RSS measurements will provide a position fix for the mobile. Due to shadow fading effects, RSS method results in large range estimation errors. The accuracy of this method can be improved by utilizing pre-measured received signal strength contour centered at the receiver [16]. A fuzzy logic algorithm was shown in [17] to be able to significantly improve the location accuracy.

\subsection{Received Signal Phase}

Signal phase is another possible geolocation metric. It is well known that with the aid of reference receivers to measure the carrier phase, differential GPS (DGPS) can improve the location accuracy from about $20 \mathrm{~m}$ to within $1 \mathrm{~m}$ compared to the standard GPS, which only uses pseudorange measurements [7] One problem associated with the phase measurements lies in the ambiguity resulted from the periodicity (with period $2 \pi$ ) of the signal phase while the standard pseudorange measurements are unambiguous. Consequently, in the DGPS, the ambiguous carrier phase measurement is used in fine-tuning the pseudorange measurement. A complementary Kalman filter is used to combine the low noise ambiguous carrier phase measurements and the unambiguous but noisier pseudorange measurements [7] For indoor geolocation system, it is possible to use the Received Signal Phase method together with TOA/TDOA or RSS method to fine-tune the location estimate. However unlike the application scenario of DGPS where LOS signal path is always observed, the 
multipath and no-line-of-sight condition of the indoor radio channel causes more errors in the phase measurements.

\section{Example Wireless Indoor Geolocation Systems}

Commercial indoor geolocation products have already appeared in the market. In this section, we present a technical overview of two example systems, Pinpoint Local Positioning System and Paltrack indoor geolocation system. Both companies claim that the indoor geolocation systems they developed can provide adequate location accuracy and location services in indoor environment.

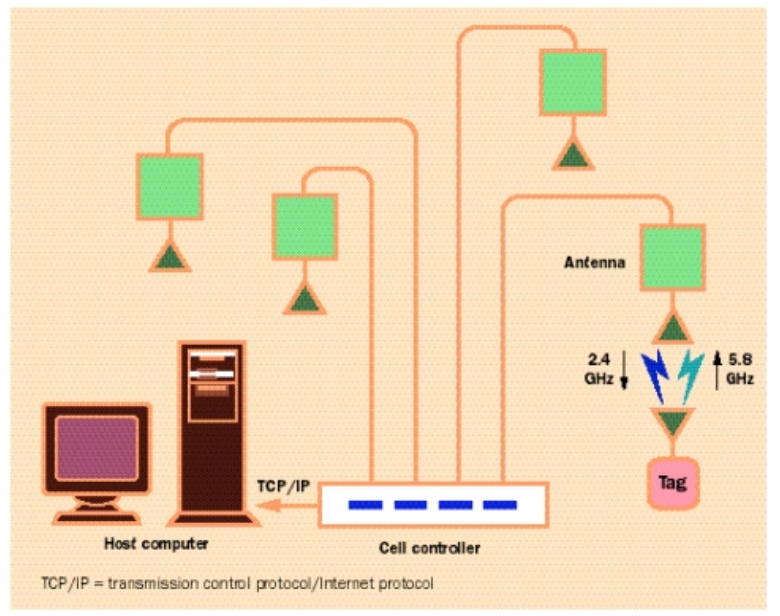

Fig. 4. PinPoint system architecture [4].

\subsection{PinPoint Local Positioning System [4]}

The system architecture of the PinPoint local position system is shown in Fig. 4. The PinPoint system uses simple-structured tags that can be attached to valuable assets or personnel badges. Indoor areas are divided into cells while each cell being served by a Cell Controller. The Cell Controller is connected to at most 16 antennas located at known positions. To locate tag position, Cell Controller transmits $2.4 \mathrm{GHz}$ spread spectrum signal through different antennas in TDD mode. Once receiving signals from the Cell Controller antenna network, tags simply change the frequency of the received signal to $5.8 \mathrm{GHz}$ and transmit back to the Cell Controller with tag ID information phase-modulated onto the signal. The distance between tag and antenna is determined by measuring round trip time of flight. With the measured distances from tag to antennas, the tag position can be obtained in the same way as in TOA method. A Host Computer is connected to Cell Controller through TCP/IP network to manage the location information of the tags. Since the Cell Controller generates the 
signal and measures round trip time of flight, there is no need to synchronize the clocks of tags and antennas.

The multipath effect is one of the limiting factors for indoor geolocation. Without multipath signal components, the time of arrival (TOA) could be easily determined from the triangular auto-correlation function of the spread spectrum signal. The triangular auto-correlation peak is two chips' (clock periods) wide at its base, and the time to rise from the noise floor to the peak is one chip. If the chipping rate were 1 $\mathrm{MHz}$, it would take $1000 \mathrm{~ns}$ to rise from the noise floor to the peak, providing a "ruler" with a thousand $30-\mathrm{cm}$ increments. A 40-MHz chipping rate was chosen for PinPoint system, providing a ruler of $25 \mathrm{~ns}$ that provides real-world increments of about 3.8 meters. Because of regulatory restrictions in the 2.44 and $5.78 \mathrm{GHz}$ bands, faster chipping rates are not easy to achieve, and signal-processing techniques must be used to further improve the accuracy. Also to minimize the multipath effect, different frequency bands are used for uplink and downlink communication to avoid interference between the channels.

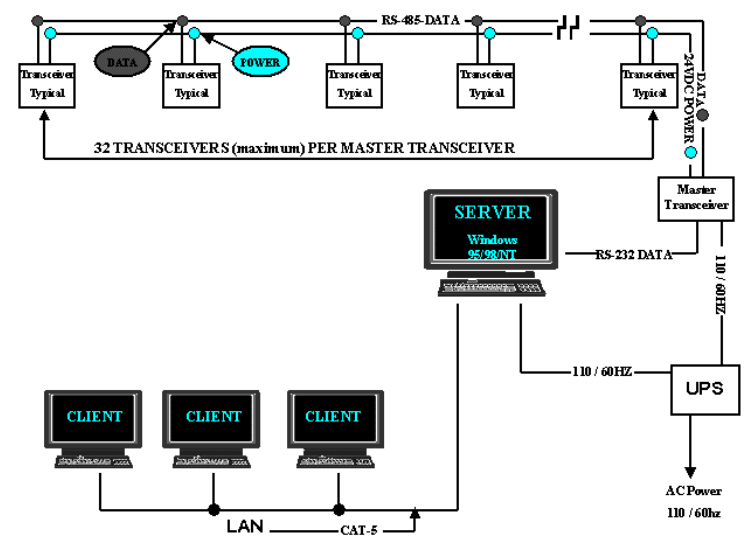

Fig. 5. Paltrack system architecture [5].

\subsection{PalTrack Indoor Geolocation System [5]}

The infrastructure of Paltrack indoor geolocation system, developed by Sovereign Technologies Corp., consists of tags, antennas, cell controllers and administrative software Server system as shown in Fig. 5. The PalTrack system utilizes a network structure that resides on an RS-485 node platform. A network of transceivers is located at known positions within the serving area while the transmitter tags are attached to assets. The tag transmitters transmit unique identification code at 418 $\mathrm{MHz}$ frequency band to a network of transceivers when on motion or at predefined time intervals. Transceivers estimates the tag location by measuring received signal strength (RSS) and utilizing a robust RSS-based algorithm patented by Sovereign Technologies Corp. The Master Transceiver collects measured information from the transceivers and relays it to a PC-based Server system. The accuracy for PalTrack is 
0.6 to $2.4 \mathrm{~m}$. The key component of the PalTrack system is the RSS-based geolocation algorithm.

\section{An Indoor Geolocation Demonstrator}

As we mentioned earlier, the indoor radio channel is very different from that of GPS systems or cellular system. To study the performance of various indoor geolocation techniques, a software indoor geolocation demonstrator is being developed at CWINS, WPI. Some general descriptions of the demonstrator as well as some simulation results will be presented in this section.

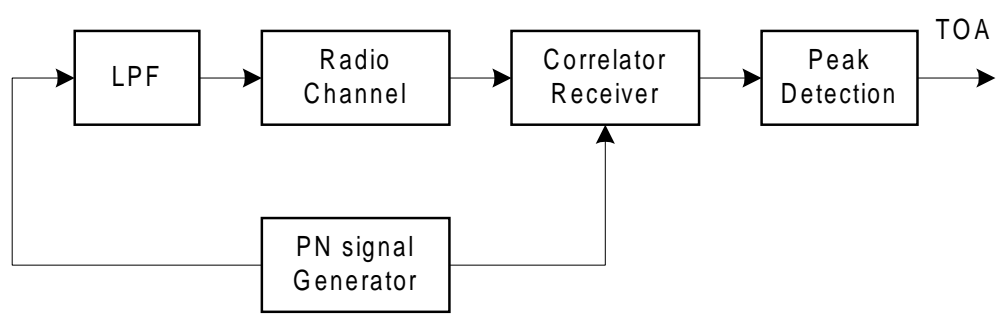

Fig. 6. Simulation of baseband DSSS geolocation system.

\subsection{DSSS Indoor Geolocation System}

The direct-sequence spread spectrum (DSSS) technique has been used in ranging systems for many years and is the principle behind GPS techniques. A block diagram of the simulated baseband DSSS geolocation system is shown in Fig. 6. For convenience, the PN (pseudo-random noise) signal generator is used in both transmitter and receiver with the assumption of time synchronization between geolocation transmitter and receiver. The lowpass filter (LPF) is used to take into account the band-limitation condition of the realistic radio transceiver systems. The autocorrelation characteristics of the PN sequence are fundamental to the distance (or TOA) estimation. A 31-chip Gold sequence was used with the chipping interval $T_{c}=25 \mathrm{~ns}$ and the sampling period $T_{s}=5 \mathrm{~ns}$ which provides a width of 50ns at the base of the triangular autocorrelation function of PN signal and an accuracy of $5 \mathrm{~ns}$ in the TOA measurements. While the multipath radio channel spreads the transmitted signal, geolocation receivers are only interested in detecting the DLOS path, i.e. determining the arrival time of the first peak in the output autocorrelation signal [1]. With TOA measurements of signals from multiple reference transmitters, the position estimate of the receiver can be obtained iteratively using nonlinear least square algorithm. 


\subsection{Channel Measurement and Ray-Tracing}

The channel profiles used in this simulation can be obtained in two ways. One is using a frequency domain measurement system described in [18]. The centerpiece in this system is a network analyzer that sweeps the channel from 900- $1100 \mathrm{MHz}$. The output signal is first amplified with an amplifier and then connected to the transmitter antenna through a long cable. The receiver antenna passes the signal through a chain of low noise amplifiers that are connected to input port of the network analyzer. The network analyzer records the frequency magnitude and phase responses of the channel. Fig. 7 shows an example of measured radio channel frequency response. For the geolocation simulation, we need to measure the actual frequency-domain channel response between the points of each reference transmitter and receiver. Then the channel impulse responses between transmitter and receiver, which we refer to as a channel time profile or simply a profile, can be obtained by taking the Fourier transform to the measured frequency channel response.

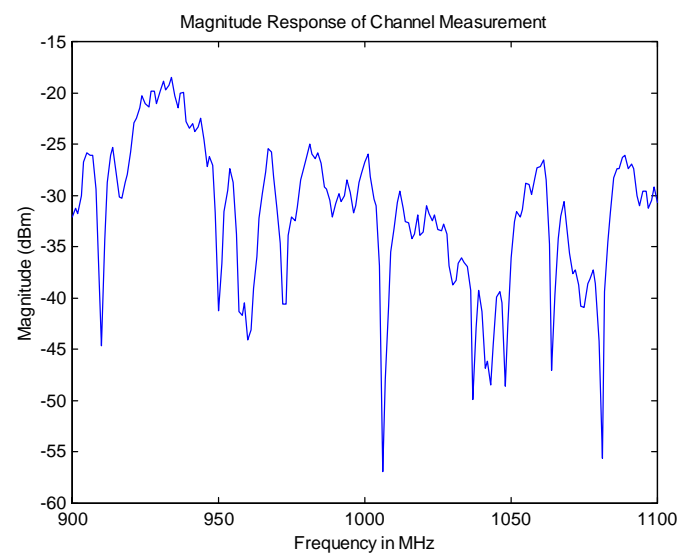

Fig. 7. Magnitude response of a frequency domain channel measurement.

Another way to obtain the channel profiles is using ray-tracing channel modeling method. Radio signals with frequencies larger than $300 \mathrm{MHz}$ have extremely small wavelengths compared to the dimensions of building features so that electromagnetic waves can be treated simply as rays [19]. This is the principle behind ray-tracing method for radio channel modeling. In our simulation, we used CWINS 2D raytracing software to obtain the channel profiles, as shown in Fig. 8, between each reference transmitter and the receiver. Then the channel profiles can be directly used in geolocation simulations to obtain TOA and distance measurements. 


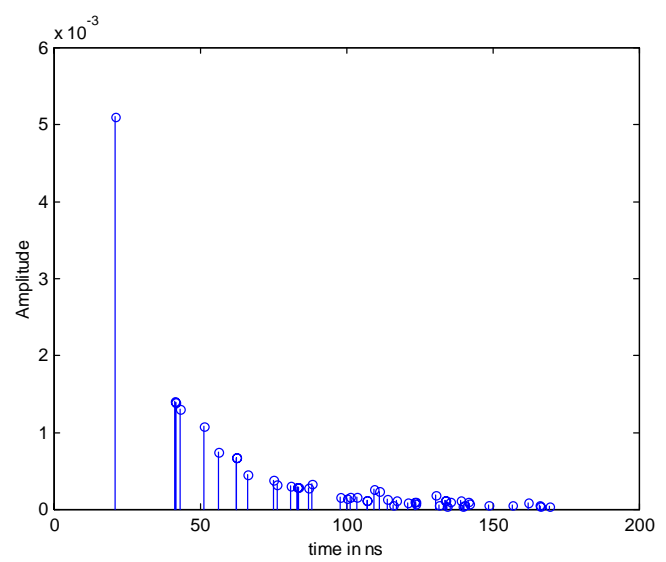

Fig. 8. Channel profiles obtained using 2-D ray-tracing software.

\subsection{Simulation Results}

A fundamental issue in geolocation is the analysis of the accuracy of positioning. For spread spectrum geolocation systems, one of the limiting factors is the available channel bandwidth, i.e. the larger the channel bandwidth, the higher the measurement resolution which closely relates to the accuracy of TOA measurement. The multipath indoor radio channel makes the analysis very complicated. This section presents results of simulations that relate the bandwidth with the accuracy of positioning.

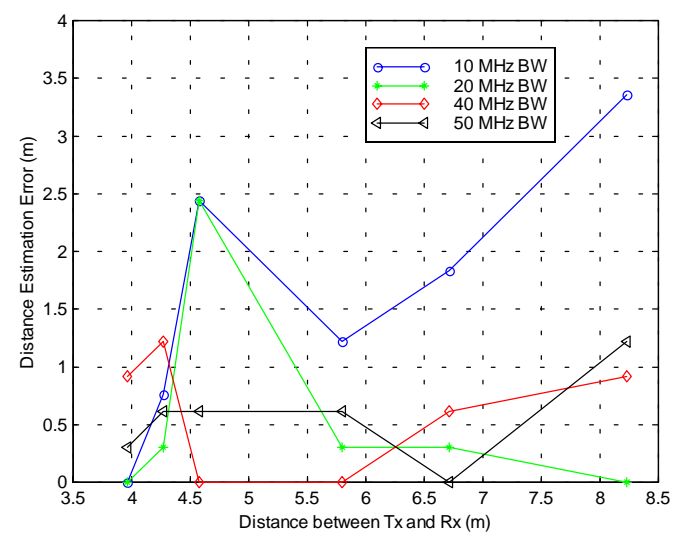

Fig. 9. Effects of channel bandwidth in ranging errors (with measurement-based method) [20].

Fig. 9 shows results obtained from channel measurement-based simulations. Table 1 shows the ranging errors between the transmitters and the receiver for different signal bandwidth obtained from both measurement based and ray-tracing based 
simulations. In Fig. 9 we observe that ranging errors are less than $3.5 \mathrm{~m}$ in all the cases. But to achieve less than $1.5 \mathrm{~m}$ accuracy, a bandwidth of larger than $20 \mathrm{MHz}$ is needed.

Table 1. Mean ranging errors using measurement based and ray-tracing based methods [20][21].

\begin{tabular}{|c|c|c|}
\hline Bandwidth (MHz) & $\begin{array}{c}\text { Measurement based } \\
\text { ranging error }(\mathrm{m})\end{array}$ & $\begin{array}{c}\text { Ray-tracing based } \\
\text { ranging error }(\mathrm{m})\end{array}$ \\
\hline 10 & 2.18 & 1.48 \\
\hline 20 & 1.10 & 0.89 \\
\hline 40 & 1.22 & 0.55 \\
\hline 50 & 1.07 & 0.32 \\
\hline
\end{tabular}

By using nonlinear least square algorithm to estimate receiver position from TOA measurements, we can employ more than two TOA measurements. Table 2 shows mean of the location estimation errors when different numbers of TOA measurements are used in the geolocation algorithm. Both measurement-based and ray-tracing based simulations show consistent results. But the ray-tracing based method is more convenient since for the measurement-based simulations, channel responses between all the transmitters and the receiver have to be measured.

Table 2. Mean location estimation errors with different numbers of TOA measurements [21].

\begin{tabular}{|c|c|c|}
\hline $\begin{array}{c}\text { Number of TOAs } \\
\text { used in geolocation }\end{array}$ & $\begin{array}{c}\text { Measurement based } \\
\text { location error }(\mathrm{m})\end{array}$ & $\begin{array}{c}\text { Ray-tracing based } \\
\text { location error }(\mathrm{m})\end{array}$ \\
\hline 3 & 1.25 & 0.81 \\
\hline 4 & 0.69 & 0.52 \\
\hline 5 & 1.05 & 0.31 \\
\hline 6 & 1.05 & 0.49 \\
\hline 7 & 1.21 & 0.39 \\
\hline
\end{tabular}

\section{Conclusions}

In this paper, we briefly reviewed various geolocation metrics and discussed usability of these metrics in indoor environment. Due to the serious multipath and no-line-ofsight propagation condition of the indoor radio channel, TOA/TDOA and Received Signal Strength methods are more appropriate than AOA method. Technical overview of two example wireless indoor geolocation products is then presented. Simulation results obtained from a software indoor geolocation testbed show that available channel bandwidth plays an important role in the accuracy of indoor geolocation systems. It was shown that ray-tracing based method provides consistent results with that of measurement-based method and the ray-tracing based method is more convenient than the frequency-domain measurement method. 


\section{Acknowledgement}

The authors would like to express their appreciation to TEKES, Nokia, and Finnish Airforce for supporting most parts of this project. We are thankful to two MQP groups at CWINS, WPI for their contributions to this work: K.H. Shah, C.M. Kelly and D.S. Hastings for measurement-based simulations and S. Dasmah, C.D. Le and T.Q. Nguyen for ray-tracing based simulations. We also thank Dr. Jacques Beneat and Dr. Prashant Krishnamurthy, our colleagues at CWINS, for fruitful discussions and a variety of help.

\section{References}

[1] K. Pahlavan, P. Krishnamurthy and J. Beneat, "Wideband radio propagation modeling for indoor geolocation applications", IEEE Comm. Magazine, pp. 60-65, April 1998.

[2] P. Krishnamurthy, K. Pahlavan, J. Beneat, "Radio propagation modeling for indoor geolocation applications", Proceedings of IEEE PIMRC'98, September 1998.

[3] Jay Werb and Colin Lanzl, "Designing a positioning system for finding things and people indoors", IEEE Spectrum, vol. 35, No. 9, Sep. 1998.

[4] PinPoint Local Positioning System, http://www.pinpointco.com/.

[5] PalTrack Tracking Systems, http://www.sovtechcorp.com/.

[6] J. Caffery, Jr. and G.L. Stuber, "Subscriber Location in CDMA Cellular Networks", IEEE Trans. Veh. Technol., vol. 47, No. 2, May 1998.

[7] E.D. Kaplan, Understanding GPS: Principles and Applications, Artech House Publishers, 1996.

[8] G. Morley and W. Grover, "Improved location estimation with pulse-ranging in presence of shadowing and multipath excess-delay effects", Electronic Letter, vol. 31, pp 16091610, Aug., 1995.

[9] G. Turin, W. Jewell and T. Johnston, "Simulation of urban vehicle-monitoring systems", IEEE Trans. Veh. Technol., vol. VT-21, pp. 9-16, Feb. 1972.

[10] H. Hashemi, "Pulse ranging radiolocation technique and its application to channel assignment in digital cellular radio", Proc. IEEE VTC'91, pp. 675-680, 1991.

[11] P. Goud, A. Sesay and M. Fattouche, "A spread spectrum radiolocation technique and its application to cellular radio", Proc. IEEE Pacific Rim Conf. Comm., Comp. and Signal processing, 1991, pp. 661-664.

[12] W.H. Foy, "Position-location solutions by Taylor-series estimation", IEEE Trans. Aerospace and Electronic Systems, vol. AES-12, pp. 187-194, Mar. 1976.

[13] D.J. Torrieri, "Statistical theory of passive location system", IEEE Trans. Aerospace and Electric Systems, vol. AES-20, No. 2, Mar. 1992.

[14] J.S. Abel and J.O.Smith, "A divide-and-conquer approach to least-squares estimation", IEEE. Trans. Aerospace and Electric Systems, vol. 26, pp. 423-427, Mar. 1990.

[15] Y.T. Chan and K.C. Ho, "A simple and efficient estimator for hyperbolic location", IEEE Trans. Signal Processing, vol. 42, No. 8, pp. 1905-1915, Aug. 1994.

[16] W. Figel, N. Shepherd and W. Trammell, "Vehicle location by a signal attenuation method", IEEE Trans. Vehicular Technology, vol. VT-18, pp. 105-110, Nov. 1969.

[17] Han-Lee Song, "Automatic Vehicle Location in Cellular Communications Systems", IEEE Trans. Vehicular Technology, vol. 43, No. 4, pp. 902-908, Nov. 1994.

[18] S.J. Howard and K. Pahlavan, "Measurement and analysis of the indoor radio channel in the frequency domain", IEEE Trans. Instr. Meas. No. 39, pp. 751-755, 1990.

[19] K. Pahlavan and A. Levesque, Wireless Information Networks, John Wiley and Sons, New York, 1995. 
[20] K.H. Shah, C.M. Kelly and D.S. Hastings, MQP Project Report: Wireless Indoor Geolocation System, CWINS, Worcester Polytechnic Institute, May 1999.

[21] S. Dasmah, C.D. Le and T.Q. Nguyen, MQP Project Report: Simulation Platform for Performance Evaluation of Indoor Geolocation, CWINS, Worcester Polytechnic Institute, January 2000. 